\title{
Ethical aspects of using radiolabelling in aerosol research
}

\section{L Everard}

\section{The potential benefits of undertaking a study must justify any potential risk}

pap his issue of Archives includes two papers assessing drug delivery systems used to administer aerosols to infants and children. ${ }^{12}$ The publication of these papers is notable for two reasons. The first, and most striking, is that they use radiolabelled aerosols and gamma scintigraphy to answer the questions posed. While there are now many such studies published in a variety of respiratory and paediatric journals, this journal has previously, on the basis of reviewers' comments, declined to publish such papers on the grounds that it would appear to be "unethical" to administer radioactivity to children for such research purposes. In addition their publication occurs at a time when most journals have, quite reasonably, become increasingly resistant to publication of studies purporting to assess inhaler systems in vitro or in vivo which do not include evidence of clinical relevance.

In most respects the ethical issues surrounding the use of radiolabelled deposition studies are no different to those involving children in other types of research. Issues surrounding the need on the one hand to act in an individual child's best interest while on the other hand protecting children from the potential harmful effects of using inadequately tested drugs, and the difficulties of obtaining truly informed consent in young children involved in pharmaceutical trials have been discussed frequently in recent years. ${ }^{3-5}$ It is recognised that such studies are vital if therapeutic advances are to be achieved, but that there are potential risks to the child participating in the study, particularly when a new chemical entity (NCE) is being assessed. In order to justify any such risk, studies must be well designed and powered in order to provide adequate answers to questions regarding safety and efficacy. In addition the potential benefits of undertaking a study must justify any potential risk. ${ }^{5}$

Radiolabelled deposition studies differ from a clinical safety/efficacy study of a NCE in two respects. Firstly, the risk associated with radiolabelled studies can be quantified and minimised by careful design. In contrast, the risk posed by an
NCE is not fully quantifiable since safety data available prior to a clinical study may be limited to animal studies and limited phase 2 studies in adults. The other important difference is that deposition studies cannot answer clinically important questions. While the information may be interesting and may modify future clinical studies, the results by themselves cannot answer fundamental questions regarding efficacy and safety. In this respect they are analogous to the phase 2 pharmacokinetic studies that precede clinical trials. For these reasons a clear understanding of the field of aerosol therapy, the options available when considering a particular question, and the limitations of deposition studies, is required when contemplating a study.

\section{RADIOLABELLED STUDIES}

Radiolabelled studies have become increasingly sophisticated and prevalent over the years. Initially undertaken by clinical scientists to obtain fundamental information that could not be obtained in other ways, such studies in adults have now become a standard part of product development, and indeed are also being undertaken as a marketing tool. There are a number of technical challenges to be addressed, including effective labelling of the drug and accurate analysis and interpretation of the data. ${ }^{5-7}$ Studies assessing solutions delivered by nebulisers such as those in the current studies are relatively straightforward in that the "soup" method appears to be adequate. This simply involves adding the label, usually $99 \mathrm{~m}$-technetium, to the solution. Studies involving dry powders, pMDIs, and suspensions are much more difficult and require extensive validation.

Various techniques are used to correct for attention, map the edge of the lungs, describe areas of interest, and analyse the data in 2D and 3D. Failure to have a common approach, as illustrated by the very different approaches used in the two current studies, means that it is not possible to compare results obtained in different centres and that results are essentially ball-park figures. For example, one study does not appeared to have corrected for attenuation of activity by the body tissue, an important step in attempting to obtain accurate values, while the other does not indicate which of many different techniques was used. While such limitations prevent direct comparisons between studies, this approach is probably valid when comparing two delivery systems in the same patient group. It should also be noted that the images obtained using gamma scintigraphy are very pretty but can be very misleading in that the appearance can be very easily and effectively manipulated to show apparently conflicting results. Therefore the data must be presented in a clear and unambiguous manner.

The greatest benefit of radiolabelling drugs is that the pattern of deposition throughout the airways can be determined, including that deposited in the upper airway and the pattern of distribution within the lungs. Information regarding the pattern of deposition within the lungs is almost impossible to obtain in other ways, though planar scintigraphy is relatively insensitive to changes of deposition within the lungs because of the complex 3D nature of the airways. Such studies do not, however, provide information regarding total body exposure to a drug for which pharmacokinetic studies are required.

\section{ASSESSMENT OF RISK}

The view of reviewers who considered that such studies are "unethical" was presumably based on a sense of unease regarding radioactivity rather than a rational exploration of risk. At the doses used in these studies it is clear that there should not be any clinical effects attributable to direct cytotoxic or "deterministic" effects since they are well below the threshold dose required to induce such effects. ${ }^{6}$ However, it is known that there is no threshold dose for so call "stochastic" effects-that is, the radiation induced changes that occur in cells exposed to radiation such as mutation or deletions of DNA. The effects of such changes may take many years to become manifest. The lack of any threshold would imply that exposure to any ionising dose might be harmful. The long latent period before manifestation of such effects means that exposure in childhood may represent a greater risk than a similar exposure in adulthood as children are likely to live longer. It is also possible that children are more sensitive to radiation and that they may pass on harmful mutations to their children, though there is no direct evidence for either of these concerns (other than possibly increased susceptibility of the juvenile thyroid). Calculating the likely risk of problems involves calculating the "effective dose", which will be dependent on the type of radiation $(\alpha, \beta$, or $\gamma)$, 
the dose used, residence time in the organ, and the type of organ(s) exposed, since different organs vary in their tolerances to radiation. The effective dose allows risk to be considered on a unified scale and can be compared to other sources, such as background or that involved in a transatlantic flight.

When administered as ${ }^{99 m}$ Tc sodium pertechnetate, it is rapidly cleared from the lung with a half life in the lungs of 10-15 minutes; clearance is still relatively rapid when administered as ${ }^{99 \mathrm{~m}} \mathrm{Tc}$ DTPA, as used in these studies, which has a residence half life of up to 150 minutes. For isotopes rapidly cleared from the lungs it appears that the greatest exposure is experienced by the bladder.

Some observers have been concerned that such estimates of risk are based on overall exposure to the organ and ignore the potential impact of relatively high doses at an individual cellular level adjacent to the sites of deposition. There has been very little work undertaken to address this specific issue, but the doses used to date have been such that radiation protection workers consider that the risk is extremely low, particularly when using formulations rapidly cleared from the lungs. The use of a low dose gamma emitter with a short radiation half life together with rapid clearance from the lungs and body results in a low effective dose equivalent to a few weeks background radiation, and would certainly appear to be less than that associated with a computed tomography scan of the chest.

Previous radiolabelled studies have undoubtedly provided insights into drug delivery to the lungs in both adults and children that would probably not been obtained in any other way. While the risk from such studies appears extremely low it cannot be said that they are without risk. Therefore in order to justify undertaking any study in children, a number of criteria should be met. This approach should only be used in children when ${ }^{6}$ :

- It is to answer an important clinical question

- The answer cannot be obtained in any other acceptable way

- The study is designed in such a way as to obtain maximum information

- The children are exposed to lowest dose consistent with obtaining high quality data.

Such criteria necessitate a thorough understanding of inhaled therapy and close collaboration with medical physicists.

\section{ASSESSMENT OF AEROSOL DELIVERY SYSTEMS}

There are a variety of in vitro and in vivo techniques that can be used to assess inhaler devices. ${ }^{6-8}$ Most are at best a guide to what might happen in real life and most have at one time or other inadvertently or, occasionally, intentionally mislead clinicians. Standard benchtop testing using techniques such as liquid impingers and laser particle sizing have a role in product development and quality control, and represent a starting point for understanding what might be happening in patients. Their attraction for some researchers is that they are quick and relatively easy to undertake, and this led to a considerable number of such publications in the late 1980s and early 1990s. Unfortunately they are so far removed from patients that they provide at best a suggestion of what is likely to happen in the real world. The in vitro assessment of devices improved significantly over the past decade, with the development of increasingly complex dynamic systems which now do provide much greater information and that appear to correlate to a greater degree with results in patients. ${ }^{9-11}$

Obtaining information regarding the deposition of drug can be obtained using classic pharmacokinetic techniques, but these do require multiple venous samples, ${ }^{72}$ always difficult in very young children, though this can be reduced by means of "population" pharmcokinetics, using fewer samples from more subjects. There are indirect pharmacokinetic approaches involving the collection of urine samples, ${ }^{913}{ }^{14}$ and this approach has been employed using sodium chromoglycate, salbutamol, and aminoglycoside antibiotics. The necessity for complete and in some cases timed urines makes the use of such techniques difficult or impossible in very young children.

\section{LIMITATIONS OF PRECLINICAL STUDIES}

In vitro and in vivo studies may provide interesting data that may allow us to try and optimise inhaled therapy but ultimately, as noted above, the only way to obtain safety and efficacy data is to undertake clinical trials. The two studies published in this issue illustrate the potential for considering alternative approaches that may obviate or minimise the need for radiolabel studies. It could reasonably argued that neither was necessary, even though they have generated interesting data. The study by Amitav et al does, however, illustrate the type of information that might be derived from deposition studies that is difficult to obtain in other ways.

The study by Byrne et al compared lung delivery using a Halolite system with the current standard delivery system in their unit, the Pari LC Plus. As noted the Halolite was preset to deliver a given dose of a suspension based formulation of budesonide, and had not been assessed for the delivery of colistin solution. A subsequent version of the device is now being marketed specifically for this purpose. A simple dynamic in vitro test utilising a breathing simulator based on the breathing patterns of children ${ }^{10}$ would almost certainly have provided the same answer regarding the relative dose delivered to the patient, and a realisation that alteration of the settings was required to achieved equivalent drug delivery (if this is desirable-see below).

The sputum studies could have been undertaken without radioactivity. However, this is not an entirely satisfactory method of assessing drug delivery since reduced lung function and to a lesser extent larger particle sizes will result in more central deposition and greater concentrations of antibiotic in the sputum collected after inhalation. Thus sputum concentrations can be misleading and certainly cannot be relied on to reflect the total lung dose. ${ }^{15}$

Data regarding the pattern of deposition in the lungs could not be obtained in other ways. Although there was a trend for improved peripheral deposition using the Halolite, the study was unable to show a significant difference in penetration index, which is not too surprising given the limitation of gamma scintigraphy and the similarity in mass median aerosol diameter of the aerosols generated by both devices.

What is the clinical significance of their findings? Their conclusions appear to be based on the assumption that more is better. The clinical evidence that colomycin is useful is based on studies of "eradication" and "maintenance" therapy of short duration. ${ }^{16}$ These studies used standard jet nebulisers whose "efficiency" is significantly lower than the Pari LC and indeed lower than that of the Halolite. While more may be better in terms of cidal concentrations there is, as with inhaled steroids, the potential for more to mean more adverse effects, ${ }^{18}$ and consequently more may actually adversely affect the "therapeutic index". The concentrations in the proximal airways achieved using both devices probably greatly exceeds the desired therapeutic concentrations. The trend towards improved peripheral deposition associated with the Halolite may or may not have clinical relevance as the drug may possibly reach organisms "deeper" in the lung. Is improved total dose better than improved peripheral deposition? Once again a "deposition" study whose implications appear straightforward throws up further questions that ultimately cannot be answered without carefully designed clinical studies.

The study by Amirav et al provides some interesting data that might be difficult to obtain in other ways. Their data appear to confirm that a hood represents a valuable alternative to a facemask 
when delivering aerosols to very young children. Prestudy data could have been obtained using a breathing simulator in conjunction with a realistic upper airways cast from an infant. ${ }^{11}$ In vitro confirmation could have been obtained using a classic or indirect pharmacokinetic study. However, this approach would not provide information regarding the pattern of deposition within the airways. Of interest in this study is the extremely high upper airways deposition when using both delivery systems, representing $76 \%$ of dose deposited in the body as a result of nose breathing. This is a much greater percentage than seen in older children and adults using a mouthpiece, but is similar to that seen in adults inhaling through their nose. ${ }^{19}$ It is also not very different to the upper airway deposition seen with a pMDI used without spacer. Such information probably could not be obtained from an indirect pharmacokinetic technique in infants, but might become evident using a classic pharmacokinetic technique. Indeed it could be argued that a pharmacokinetic study would be more valuable in that it would provide information regarding the total body exposure resulting from this pattern of deposition which is clearly of relevance for drugs such as inhaled corticosteroids. Moreover it is not entirely original with a number of previous studies indicating that upper airways deposition is very high when infants and young children inhale through their nose. ${ }^{20-22}$

Were the studies unethical? In neither case is it likely that the subjects were harmed since the risk, though finite, is extremely low. Information relevant to the questions posed could have been obtained in other ways in both studies, and indeed for the study by Byrne et al, would probably have been just as informative. The argument for the use of hoods as an alternative to a facemask was greatly strengthened by the imaging data obtained by Amirav et al. In this case the images (with their accompanying data) are very persuasive and it would be difficult to provide such convincing data in other ways. This paper did also include data suggesting clinical benefit, though this was not a placebo controlled study.

\section{SUMMARY}

Radiolabelled pulmonary deposition studies in children can be undertaken using very low doses carrying apparently very low risk. For studies using solutions delivered by nebulisers, they are relatively simple and straightforward to undertake, making them attractive for researchers. However, before resorting to such studies the question to be answered should be carefully formulated. It should be clear that it cannot be answered effectively in other ways and that the study has been designed by a team who understand the strengths and limitations of the various possible approaches to assessing inhaled devices and which include radiation protection expertise. These studies do not replace the need for careful clinical trials since they cannot answer questions regarding safety and efficacy.

Arch Dis Child 2003;88:659-661

Correspondence to: Dr M L Everard, Dept of Respiratory Medicine, Sheffield Children's Hospital, Western Bank, Sheffield S10 2TH, UK. M.I.everard@sheffield.ac.uk

\section{REFERENCES}

1 Byrne NM, Keavey PM, Perry JD, et al. Comparison of lung deposition of colomycin using the HaloLite and the Pari LC Plus nebulisers in patients with cystic fibrosis. Arch Dis Child 2003;88:715-8.

2 Amirav I, Balanov I, Gorenberg $M$, et al. Nebuliser hood compared to mask in wheezy infants: aerosol therapy without tears! Arch Dis Child 2003;88:719-23.

3 Royal College of Paediatrics and Child Health, Ethics Advisory Committee. Guidelines for the ethical conduct of medical research involving children. Arch Dis Child 2000;82:177-82

4 Stephenson T. New medicines for children who is protecting the rights of the child. Curr Paediatr 2002:12:331-5.

5 Sutcliffe AG. Testing new pharmaceutical products in children. BM 2003;326:64-5.

6 Everard ML. The use of radiolabelled aerosols for research purposes in paediatric patients: ethical and practical aspects. Thorax 1994;49:1259-66.
7 Everard $M L$, Dolovich $M B$. In vivo measurements of lung dose. In: Bisgaard $\mathrm{H}_{\text {, }}$ O'Callaghan, C, Smaldone G, eds. Drug delivery to the lung: clinical aspects. Lung biology in health and disease series. New York: Marcel Decker, 2001.

8 Everard ML, Le Souef PN. Aerosol therapy and delivery systems. In: Taussig L, Landau L, eds. Textbook of paediatric respiratory medicine. Stm Louis, MO: Mosby, 1998

9 Everard ML, Clark AR, Milner AD. Drug delivery from holding chambers with attached facemask. Arch Dis Child 1992;67:580-5.

10 Nikander K, Denyer J, Everard ML, et al. In vitro determination of the inhaled mass of drug can replace in vivo measurements. $J$ Aerosol Med 2000;13:139-46.

11 Janssens HM, de Jongste JC, Fokkens WJ, et al. The Sophia anatomical infant nose-throat (Saint) model: a valuable tool to study aerosol deposition in infants. J Aerosol Med 2001; 14:433-41.

12 Agertoft L, Andersen A, Weibull E, et al. Systemic availability and pharmacokinetics of nebulised budesonide in preschool children. Arch Dis Child 1999;80:241-7.

13 Iles R, Lister P, Edmunds AT. Crying significantly reduces absorption of aerosolised drug in infants. Arch Dis Child 1999;81:163-5.

14 Hindle $M$, Chrystyn $H$. Determination of the relative bioavailability of salbutamol to the lung following inhalation. Br J Clin Pharmacol 1992;34:311-15.

15 Ilowite JS, Gorvoy JD, Smaldone GC. Quantitative deposition of aerosolized gentamicin in cystic fibrosis. Am Rev Respir Dis 1987; 136:1445-9.

16 Littlewood JM, Smye SW, Cunliffe H. Aerosol antibiotic treatment in cystic fibrosis. Arch Dis Child 1993;68:788-92.

17 Frederiksen B, Koch C, Hoiby N. Antibiotic treatment of initial colonisation of Pseudomonas aeruginosa postpones chronic infection and prevents deterioration of pulmonary function in cystic fibrosis. Pediatr Pulmonol 1997;23:330-5

18 Jones A, Elphick H, Pettitt E, et al. Colistin stimulates the activity of neutrophil elastase and Pseudomonas aeruginosa elastase. Eur Respir J 2002; 19: 1 136-41.

19 Everard ML, Hardy JG, Milner AD. Comparison of nebulised aerosol deposition in the lungs of health adults following oral and nasal inhalation. Thorax 1993;48: 1045-6.

20 Murakam G, Igarashi T, Adachi Y. Measurement of bronchial hyperactivity in infants and preschool children using a new method. Ann Allergy 1990;64:383-7.

21 Chua HL, Collis GC, Newbury AM, et al. The influence of age on aerosol deposition in children with cystic fibrosis. Eur Respir $J$ 1994:7:2185-91.

22 Tal A, Golan H, Graver N, et al. Deposition pattern of radiolabeled salbutamol inhaled from a metered-dose inhaler by means of a spacer with mask in young children with airway obstruction. J Pediatr 1996; 128:479-84. 